\title{
An in vitro Model for Blood Brain Barrier Permeation
}

\author{
R. Bauer ${ }^{1}$, R. Lauer ${ }^{1}$, B. Linz ${ }^{2}$, F. Pittner ${ }^{3}$, G.A. Peschek ${ }^{4}$, G.F. Ecker ${ }^{1}$, P. Friedl ${ }^{5}$, C.R. Noe ${ }^{1, *}$ \\ ${ }^{1}$ Institute of Pharmaceutical Chemistry, University of Vienna, A-1090 Wien, Austria \\ ${ }^{2}$ Institute of Pharmaceutical Chemistry, Johann-Wolfgang-Goethe University, Frankfurt/Main, Germany \\ ${ }^{3}$ Institute of Biochemistry and Molecular Cell Biology, University of Vienna, A-1030 Wien, Austria \\ ${ }^{4}$ Institute of Physical Chemistry, University of Vienna, A-1090 Wien, Austria \\ ${ }^{5}$ Institute of Biochemistry, Technical University Darmstadt, D-64287 Darmstadt, Germany
}

\section{Key Words}

Blood brain barrier, in vitro model, immortalized porcine brain microvascular endothelial cells

\begin{abstract}
The ability to permeate accross the blood brain barrier (BBB) is essential for drugs acting on the central nervous system (CNS). Thus, systems that allow rapid and inexpensive screening of the BBB-permeability properties of novel lead compounds are of great importance for speeding up the drug discovery process in the CNS-area. We used immortalized porcine brain microvessel endothelial cells (PBMEC/C1-2) to develop a model for measurement of blood-brain barrier permeation of CNS active drugs. Investigation of different cell culture conditions showed, that a system using C6 astrocyte glioma conditioned medium and addition of a cyclic AMP analog in combination with a type IV phosphodiesterase inhibitor (RO20-1724) leads to cell layers with transendothelial electrical resistance values up to $300 \Omega . \mathrm{cm}^{2}$. Permeability studies with U$\left[{ }^{14} \mathrm{C}\right]$ sucrose gave a permeability coefficient $P_{e}$ of $3.24 \pm 0.14 \times 10^{-4} \mathrm{~cm} / \mathrm{min}$, which is in good agreement to published values and thus indicates the formation of tight junctions in vitro.
\end{abstract}

\section{Introduction}

The ability to permeate across the blood brain barrier (BBB) is essential for drugs acting on the central nervous system (CNS). The transfer of compounds from the blood into the brain interstitial fluid is regulated by the function of brain capillary endothelial cells. Unlike peripheral endothelium, brain microvessel endothelial cells are characterized by the presence of tight intercellular junctions, 
clearance curve of the PBMEC/C1-2 culture was denoted $\mathrm{PS}_{\mathrm{c}}$, where PS is the product of permeability $\mathrm{x}$ surface area given in $\mu \mathrm{l} / \mathrm{min}$ (or $10^{-3} \mathrm{~cm}^{3} / \mathrm{min}$ ). The slope of the clearance curve for the control filter was denoted $\mathrm{PS}_{\mathrm{f}}$. The PS value for the endothelial cell monolayer $\mathrm{PS}_{\mathrm{e}}$ was calculated from: $1 / \mathrm{PS}_{\mathrm{e}}=1 / \mathrm{PS}_{\mathrm{c}}-1 / \mathrm{PS}_{\mathrm{f}}$

The $\mathrm{PS}_{\mathrm{e}}$ values were divided by the surface area of the Falcon BIOCOAT ${ }^{\mathrm{TM}}$ culture inserts $(4,2$ $\mathrm{cm}^{2}$ ) to generate the endothelial permeability coefficient $P_{e}$, in centimeters per minute.

\section{Results and Discussion}

For our studies the immortalized porcine microvascular endothelial cell line PBMEC/C1-2 previously reported by one of the authors ${ }^{6}$ was used. Cells were cultered on collagen coated Falcon BIOCOAT ${ }^{\mathrm{TM}}$ six- well membrane inserts and the transendothelial electrical resistance (TEER) was used as a measure for the state of tight junction formation which in effect restricts permeability. For in vivo conditions, TEER values in the range of $8000 \Omega \mathrm{cm}^{2}$ were estimated by Smith and Rapoport, " whereas several other authors report values in the range of $1000-2000 \Omega \mathrm{cm}^{2}{ }^{12}$ For in vitro, maximum TEER values of $150-700 \Omega \mathrm{cm}^{2}$ are reported. ${ }^{12,13}$ For development of a BBB model for routine application, several parameters needed to be determined and optimized.

\section{Influence of seeding density on the TEER value}

Rubin et al. suggested a density of 36.000 cells $/ \mathrm{cm}^{2}$ for porcine brain endothelial cells. ${ }^{13}$ Thus, cells were plated at a seeding density of 40.000 cells $/ \mathrm{cm}^{2}$ and $80.000 \mathrm{cells} / \mathrm{cm}^{2}$ and grown in conditioned medium as described above. The time course of the respective TEER values were measured. As shown in Figures $1 \mathrm{a}$ and $1 \mathrm{~b}$, a seeding density of $80.000 \mathrm{cells} / \mathrm{cm}^{2}$ leads to slightly improved TEER values and remarkably higher reproducibility (6 independent experiments). Higher seeding densities (up to 250.000 cells $/ \mathrm{cm}^{2}$ ) did not lead to a further improvement of both TEER and reproducibility.

\section{Influence of different culture conditions}

The positive effect of factors secreted by astrocytes on the barrier tightening of brain endothelial cells is well documented. ${ }^{12,14,15}$ Thus, several coculture-based systems are reported. To avoid the experimental problems of cocultures but still achieve the positive effects of astrocytes, astrocyte conditioned medium (ACM) can be used as an alternative. ${ }^{12}$ Figure 2 shows the time course of the TEER for cells grown solely in ACM obtained from rat C6-glioma cells vs. those for cells grown in 
standard medium and plated in ACM medium. This demonstrates, that cells grown solely in ACM both reach higher TEER values and maintain this level for a longer period of time, which is beneficial for permeability assays.

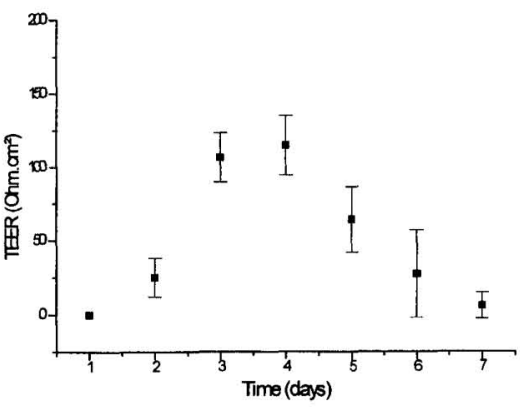

Figure 1a: Time course of the transendothelial electrical resistance applying a seeding density of $40000 \mathrm{cells} / \mathrm{cm}^{2}$

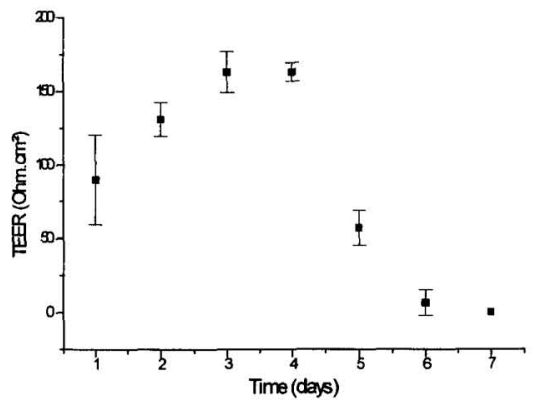

Figure 1b: Time course of the transendothelial electrical resistance applying a seeding density of $80000 \mathrm{cells} / \mathrm{cm}^{2}$

Although a coculture with rat C6-glioma cells at the bottom of the well plates led to TEER values up to $275 \Omega \mathrm{cm}^{2}$, this setting showed low reproducibility with standard deviations in the range of $153 \Omega \mathrm{cm}^{2}$. This seems to be due to the fast and unpredictable growth of the C6-glioma cells, which may lead to early depletion of important medium supplements. On basis of immunocytochemical methods Rubin et al. ${ }^{13}$ showed, that the addition of cyclic AMP or an cAMP analog in combination with a type IV phosphodiesterase inhibitor (RO20-1724) to the ACM nutrient medium enhances the expression of proteins related to tight junction formation. This led to increased TEER values of the respective cell layers up to $700 \Omega \mathrm{cm}^{2}$ in bovine brain endothelial cells. Thus, the effect of addition of 8-(4-chlorophenylthio)adenosine 3':5'-cyclic monophosphate (CPTcAMP) and RO20-1724 on PBMEC/C1-2 cells was studied. This setting led to a further increase of TEER values up to 300 $\Omega \mathrm{cm}^{2}$ (Table 1).

Figure 3 summarizes the results of the studies for optimization of the culture conditions. Application of ACM, combined with the addition of CPTcAMP and RO20-1724, results in TEER values of 250-300 $\Omega \mathrm{cm}^{2}$, which is superior to all other methods tested. 
Table 1: Transendothelial resistance values of $\mathrm{PBMEC} / \mathrm{C} 1-2$ cell layers obtained under different culture conditions

\begin{tabular}{ll}
\hline Culture Conditions & TEER $\left(\Omega . \mathrm{cm}^{2}\right)$ \\
\hline PBMEC medium & $68.6 \pm 9.7$ \\
Coculture with C6 glioma cells & $151.2 \pm 123.8$ \\
PBMEC cells grown in ACM/PBMEC medium after plating & $155.2 \pm 36.3$ \\
PBMEC cells grown solely in ACM/PBMEC medium & $234.5 \pm 31.5$ \\
Addition of CPTcAMP and RO20-1724 & $288.4 \pm 12.8$ \\
\hline
\end{tabular}

\section{Transendothelial permeability studies}

Since low permeability for substances that do not pass the blood-brain barrier in vivo is also a necessary property of an effective in vitro model, the permeability for $\mathrm{U}-\left[{ }^{14} \mathrm{C}\right]$ sucrose was determined. The cells were cultivated following the protocol determined as being optimal. Generally, layers with TEER values above $250 \Omega \mathrm{cm}^{2}$ were used for permeability assays.
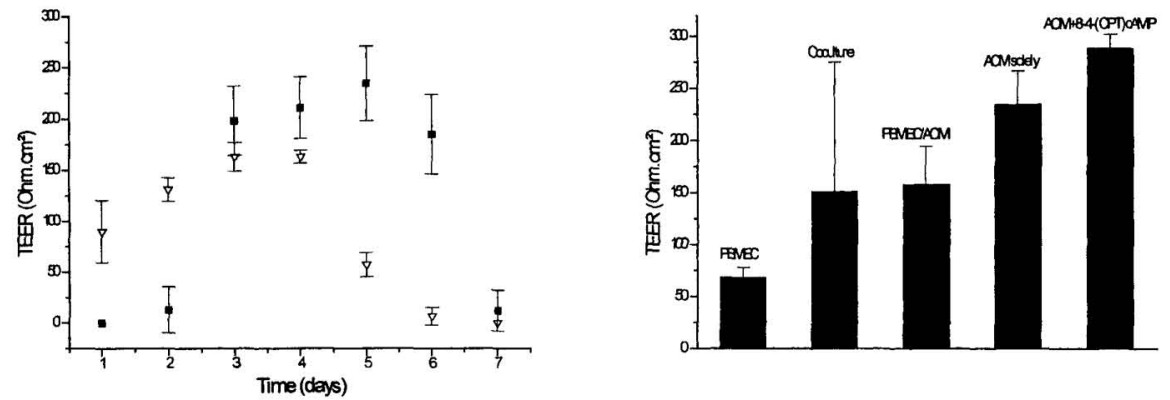

Figure 2: Time course of transendothelial electrical Figure 3: Comparison of transendothelial electrical resistance for PBMEC/C1-2 cells cultivated in different resistance for cell cultures grown under different media applications; filled squares represent cells grown cultivating methods (six independent experiments each) solely in ACM, open down triangles cells grown in PBMEC medium and plated in ACM 
To calculate permeability, the in vivo concentration of sucrose $(1 \mathrm{mg} / \mathrm{l})$ was used. To obtain a concentration-dependent transport parameter, the clearance principle was used. ${ }^{10}$ During the 40 minutes experiment, the clearance volume increased linearly with time. Figure 4 shows the clearance curves for the cell layers and the control.

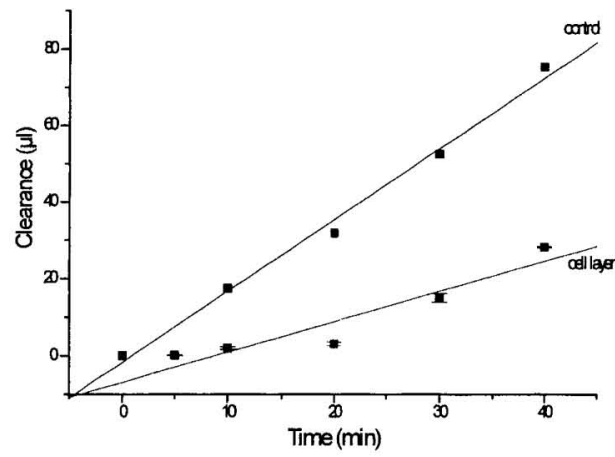

Figure 4: Clearance curve for the permeation of radiolabeled sucrose through the $\mathrm{PBMEC} / \mathrm{C} 1-2$ cell layer (each data point represents six independent experiments). Clearance (cell layer) $=0.78( \pm 0.16)$ Time $6.88( \pm 3.84) ; \mathrm{P}_{\mathrm{e}}=3.24( \pm 0.14) \times 10^{-4}$ $\mathrm{cm} / \mathrm{min}$

The endothelial permeability coefficients $P_{e}$ of the cell layers were calculated as $3.24 \pm 0.14 \times 10^{-4}$ $\mathrm{cm} / \mathrm{min}$. This value is 77 fold higher than the in vivo values for brain microvessel endothelial cells, ${ }^{4}$ but is in good agreement with other in vitro models of the blood brain barrier that have been studied $\left(6.3 \times 10^{-4} \mathrm{~cm} / \mathrm{min}\right){ }^{16}$

The results of our study demonstrate, that immortalized porcine PBMEC/C1-2 cells are a valuable tool for establishing an in vitro model of the blood brain barrier. Future work will focus on characterization of transport systems and on a further improvement of the model for analysis of highly lipophilic compounds, such as benzodiazepines.

\section{Acknowledgement}

We gratefully acknowledge the financial support provided by the Austrian Science Fund (project \# P-14582CHE). 


\section{References}

1 Joo F. (1996), Prog. Neurobiol. 48: 255

2 Pardridge W.M. (1983), Physiol. Rev. 63: 1481

3 Pardridge W.M, Blood-brain barrier methodology and biology; in: Pardridge W.M. (ed.): Introduction to the Blood-Brain Barrier, Cambridge University Press, Cambridge 1998, pp. $1-8$.

4 Pardridge W.M. (1999), J. NeuroVirology 5: 556

5 Muruganandam A., Moorhouse L., Monette R., Durkin J.P., Stanimirovic D.B. (1997), FASEB J. 11: 1187

6 Teifel M., Friedl P. (1996), Exp. Cell Res. 228: 50

7 Buchstaller H.P., Siebert C.D., Lyssy R.H., Ecker G., Krug M., Berger M.L., Gottschlich R., Noe C.R. (2000), Sci. Pharm. 68: 3

8 Berger M.L., Schödl C., Noe C.R. (1998), Eur. J. Med. Chem. 33: 3

9 Benda P., Lightbody J., Sato G., Levine L., Sweet W. (1968), Science 161: 370

10 Siflinger-Birnboim A., Del Vecchio P.J., Cooper J.A., Blumenstock F.A., Shepard J.N., Malik A.B. (1987), J. Cell Physiol. 132: 111

11 Smith Q.R., Rapoport S.I. (1986), J. Neurochem. 46: 1732

12 Raub T.J., Kuentzel S.L., Sawada G.A. (1992), Exp. Cell Res. 199: 330

13 Rubin L.L., Hall D.E., Porter S., Barbu K., Cannon C. (1991), J. Cell Biol. 115: 1725

14 Tao-Cheng J.H., Nagy Z., Brightman M.W. (1987), J. Neurosci. 7: 3293

15 Abbott N.J., Revest P.A. (1991), Cerebrovasc. Brain Metabol. Rev. 3: 39.

16 Dehouck M.P., Jolliet-Riant P., Bree F., Fruchart J.C., Cecchelli R., Tillement J.P. (1992), J. Neurochem. 58: 1790 